\title{
Acute Anterolateral Myocardial Infarction by ECG Finding
}

National Cancer Institute

\section{Source}

National Cancer Institute. Acute Anterolateral Myocardial Infarction by ECG Finding. NCI

Thesaurus. Code C102591.

An electrocardiographic finding of patholog ic Q waves with accompanying ST elevation in leads V3 through V6, which is suggestive of acute myocardial infarction of the anterolateral wall of the left ventricle. (CDISC) 